\title{
Interaction Forces on Oscillating and Translating Two Cylinders Submerged under a Free Surface
}

\author{
by Masashi Kashiwagi*, Member \\ Kamlesh Varyani**, Member
}

\begin{abstract}
Summary
With attention focused on the effects of forward speed on hydrodynamic interactions, radiation and diffraction problems are studied for submerged two circular cylinders moving with forward velocity in head and following waves. Under a linear assumption, the velocity potential is exactly obtained from the boundary-integral-equation on the body surface, using a numerical technique. The added-mass and damping coefficients, exciting forces, resultant cylinder motions, and added resistance are computed and then the hydrodynamic interaction effects on these quantities are discussed.

In the frequencies lower than $\tau=U \omega / g=1 / 4$ (where $U$ is forward speed, $\omega$ oscillation frequency, and $g$ gravity acceleration), the effects of hydrodynamic interaction on radiation forces are great, particularly for the upstream cylinder. However for $\tau>1 / 4$ the effects on the upstream cylinder are markedly reduced, but those on the downstream cylinder still exist. The interaction effects on exciting forces are small compared with radiation forces. The added resistance is approximately zero in head waves, whereas in following waves it is drastically changed by the interaction effects, while keeping the positive value for $U / C<1 / 2$ and the negative value for $1 / 2<U / C<1$ (where $C$ is phase velocity of incident wave). These numerical results are explained qualitatively by the information of the diffraction characteristics of a single circular cylinder.
\end{abstract}

\section{Introduction}

In the field of offshore engineering, one of the most attractive as well as important technical themes is the prediction of hydrodynamic forces act. ing on an offshore structure oscillating in waves. In general an offshore structure consists of several members such as bracings, columms, and lowerhulls. Thus, the hydrodynamically interacting phenomena among those members should be taken into account for the accurate prediction of hydrodynamic forces. As seen in recent progress by Kagemoto \& $\mathrm{Yue}^{1)}$, almost all the work on the hydrodynamic interaction are concerned with multiple three-dimensional bodies with vertical axisymmetry and no forward speed. However in the case of nonzero forward speed, or equivalently when the current exists, the interaction forces are expected to be influenced by the presence of forward speed. (Hereafter the term forward-speed effect will

* Research Institute for Applied Mechanics, Kyushu University

** Graduate School, Department of Naval Architecture, Kyushu University be used to include the equivalent current effect.)

The study of forward-speed effects on hydrodynamic forces is a practically important theme in ship hydrodynamics. With the fact that ships are geometrically slender, a large number of studies so far were based on the slender-body theory. Therefore various theories established are not immediately applicable to the estimation of forwardspeed effects on interaction forces acting on an offshore structure.

In our earlier work ${ }^{2)}$ ), in order to obtain a better understanding of the forward-speed effects on unsteady hydrodynamic forces, a two-dimensional problem was treated, where a cylinder moves with constant velocity, while oscillating sinusoidally un. der a free surface. In this two-dimensional problem, we could clarify with relative ease the characteristics of speed-modified waves generated on the free surface and the degree of contribution of individual wave components to the hydrodynamic forces.

In this paper, a fundamental investigation into the effects of forward speed on interaction forces is made by considering the two-dimensional prob. lem of the kind mentioned above : submerged two 
cylinders moving with forward speed and oscillating under the effect of incident wave. These two cylinders may be viewed as a simple model of lower-hulls of an offshore structure. For the zerospeed problem of interacting two cylinders, Ohku$\mathrm{su}^{4)}$ used the 'wide-spacing' approximation, based on the assumption that the bodies are far enough apart to neglect evanescent waves, with considerable success. A practical advantage of this approximation is that it enables one to predict interaction effects if only the diffraction characteristics of single body are known. In principle Ohkusu's approach can be applied also to the forward-speed problem. However in general since an oscillating and translating body generates four waves of different wavenumbers, an analytical description for multiple wave reflections will be of considerable complication. When the number of interacting bodies is relatively small, the exact calculation for all bodies as a unit seems more effective than the wide-spacing approximation. With this reason, we performed in this paper exact computations based on the boundary-integral-equation method. Then the numerical results were discussed with the speculation that only the outgoing waves contribute to the interaction forces.

\section{Problem formulation}

As shown in Fig. 1, we consider the two identical circular cylinders of radius $a$, submerged under a free surface at depth $d$. These two cylinders are assumed to be rigidly connected with separation distance $2 L$ (but the connecting member is assumed not to disturb the flow) and to move with constant forward velocity $U$, while performing small harmonic oscillations of frequency $\omega$ in surge, heave, and pitch about the midpoint of connecting member. A Cartesian coordinate system moving with the same speed as the body is employed, where the $x$-axis is positive in the direction of the body's forward motion and the $y$-axis positive downward with $y=0$ taken as the mean position of the free surface.

The formulation of the present problem is almost the same as that for a single cylinder described in reference 2). For convenience in subsequent discussions, we briefly describe here the mathematical formulation.

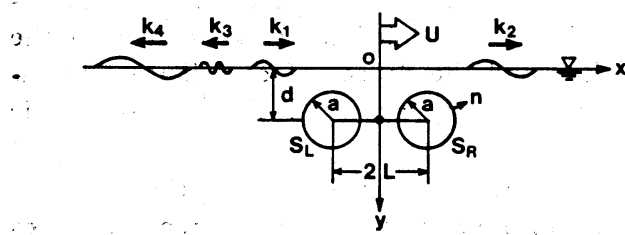

- Fig. 1 Coordinate system and schematic representation of radiated waves
With the linear assumption, we decompose the total velocity potential, satisfying Laplace's equation, in the form

$$
\Phi(x, y, t)=U\left\{-x+\varphi_{s}(x, y)\right\}+R_{e}\left\{\phi(x, y) e^{i \alpha^{\prime}}\right\}
$$

Here $\varphi_{s}$ is the steady-state perturbation potential and $\phi$ the unsteady potential with the time dependence factored out. Furthermore we express $\phi$ in the linearly decomposed form as

$$
\begin{aligned}
& \phi(x, y)=\frac{g A}{i \omega_{0}}\left\{\varphi_{I}(x, y)+\varphi_{D}(x, y)\right\} \\
& \quad+\sum_{j=1}^{s} i \omega \epsilon_{j} X_{j} a\left\{\varphi_{j}(x, y)+\frac{U}{i \omega a} \hat{\varphi}_{j}(x, y)\right\}
\end{aligned}
$$

where $\varphi_{I}$ and $\varphi_{D}$ are respectively the incident-wave and diffraction potentials, with $A$ being the amplitude and $\omega_{0}$ the frequency of the incident wave. $\epsilon_{j} X_{j}$ denotes the amplitude of $j$-th mode of motion ( $j=1$ for surge, $j=2$ for heave, $j=3$ for pitch) with the following convention

$$
\epsilon_{1}=\epsilon_{2}=1, \epsilon_{3}=L
$$

The body boundary conditions to be satisfied by the unsteady potential are summarized as follows :

$$
[H]_{R} \quad \frac{\partial \varphi_{j}}{\partial n}=n_{j}, \frac{\partial \hat{\varphi}_{j}}{\partial n}=m_{j} \quad(j=1,2,3)
$$

$$
\begin{aligned}
& \text { where } \quad m_{1} \boldsymbol{i}+m_{2} \boldsymbol{j}=-(\boldsymbol{n} \cdot \boldsymbol{\nabla}) \boldsymbol{V} \\
& \left.\begin{array}{l}
m_{8} k=-(\boldsymbol{n} \cdot \nabla)(\boldsymbol{r} \times \boldsymbol{V}) \\
n_{1} \boldsymbol{i}+n_{2} j=n
\end{array}\right\} \\
& \boldsymbol{V}=\nabla\left\{-x+\varphi_{s}(x, y)\right\} \\
& {[H]_{D} \quad \frac{\partial \varphi_{D}}{\partial n}=-\frac{\partial \varphi_{I}}{\partial n}}
\end{aligned}
$$$$
\text { where } \quad \varphi_{I}=e^{-k y \pm \imath k x}, k=\omega_{0}{ }^{2} / g
$$

The radiation potentials $\varphi_{j}, \hat{\varphi}_{3}$ and the diffraction potential $\varphi_{D}$ are subject to the free-surface condition of the form

$$
\begin{gathered}
{[F]\left[\left(i \omega-U \frac{\partial}{\partial x}\right)^{2}-g \frac{\partial}{\partial y}\right.} \\
\left.+\mu\left(i \omega-U \frac{\partial}{\partial x}\right)\right] \phi(x, y)=0 \\
\text { on } y=0
\end{gathered}
$$

Here an artificial Rayleigh viscosity $\mu$ is introduced to satisfy the radiation condition at infinity.

For infinite depth, the boundary-value problem is completed provided the following bottom condition is imposed

$$
\text { [B] } \quad \nabla \phi(x, y) \rightarrow 0 \quad \text { as } y \rightarrow \infty
$$

We note that in the body boundary condition for the radiation problem the so-called $m$-vector is included to take into account the effect of steady perturbation velocity on the unsteady flow field. In this paper, with the restriction of relatively deep submergence, an analytical solution in an infinite fluid is used for the $\varphi_{s}$-problem. Then the calculation of $m_{j}$ can be readily performed, as shown in Appendix. 


\section{Integral equation and characteristics of radiated waves}

We introduce the Green function which satisfies Laplace's equation, free-surface condition, radiation condition, and the condition of vanishing at $y=\infty$. This function can be expressed in the following form

$$
\begin{aligned}
& G(P, Q)=-\frac{1}{2 \pi}\left[\log \frac{r}{r_{1}}+\hat{G}(x-\xi, y+\eta)\right] \\
& \hat{G}(x, y)=\frac{K_{0}}{k_{1}-k_{2}}\left\{S_{1}(x, y)-S_{2}(x, y)\right\} \\
& +\frac{K_{0}}{k_{3}-k_{4}}-\left\{S_{3}(x, y)-S_{4}(x, y)\right\} \\
& S_{j}=e^{-k_{i} z}\left\{E_{1}\left(-k_{j} z\right) \pm 2 \pi i u\left(\mp I_{m} k_{j} z\right) u(1-2 \tau)\right\} \\
& j=\left(\begin{array}{l}
1 \\
2
\end{array}\right) \\
& S_{j}=e^{-k_{j} \bar{z}}\left\{E_{1}\left(-k_{j} \bar{z}\right)-2 \pi i u(-x)\right\} \quad j=3,4 \\
& \text { where } \left.\quad \begin{array}{l}
k_{1} \\
k_{2}
\end{array}\right\}=\frac{K_{0}}{2}[1-2 \tau \pm \sqrt{1-4 \tau}] \\
& \left.\left.\begin{array}{l}
k_{3} \\
k_{4}
\end{array}\right\}=\frac{K_{0}}{2}[1+2 \tau \pm \sqrt{1+4 \tau}]\right\} \\
& \left.P=(x, y), \quad Q=(\xi, \eta), \begin{array}{c}
r \\
r_{1}
\end{array}\right\}=\sqrt{(x-\xi)^{2}+(y \mp \eta)^{2}} \\
& \left.K_{0}=\frac{g}{U^{2}}, \quad \tau=\frac{U \omega}{g}, \begin{array}{l}
z \\
\bar{z}
\end{array}\right\}=y \pm i x
\end{aligned}
$$

In Eqs. (13) and (14), $u(x)$ is Heaviside's unit function and $E_{1}(z)$ the exponential integral of complex argument.

If Green's theorem is applied to this Green function and the velocity potential to be solved, the following equation can be derived, with the field point $P=(x, y)$ situated on the body surface

$$
\begin{gathered}
\frac{1}{2} \phi(P)-\left[\int_{S_{L}}+\int_{S_{R}}\right] \phi(Q) \frac{\partial}{\partial n} G(P, Q) d l \\
=-\left[\int_{S_{L}}+\int_{S_{R}}\right] \frac{\partial \phi(Q)}{\partial n} G(P, Q) d l
\end{gathered}
$$

where $S_{L}$ and $S_{R}$ denote the body surfaces of left and right cylinders, respectively (see Fig. 1).

The normal derivative $\partial \phi / \partial n$ on the right-hand side is given by the body boundary condition, so that Eq. (16) is the integral equation for the velocity potential on the body surface.

To examine the characteristics of radiated waves at infinity, let us calculate the far-field wave elevation. This can be obtained by taking into account that the exponential integral has no contribution for large values of the argument, with the result

$$
\begin{gathered}
\zeta(x)=\frac{1}{g}\left(i \omega-U \frac{\partial}{\partial x}\right) \phi(x, 0) \\
\sim \begin{cases}A_{2} e^{-i k_{2} x} & \text { as } x \rightarrow+\infty \\
A_{1} e^{-i k_{1} x}+A_{3} e^{i k_{3} x}+A_{4} e^{i k_{4} x} & \text { as } x \rightarrow-\infty\end{cases}
\end{gathered}
$$

where

$$
A_{\jmath}= \begin{cases}-\sqrt{\frac{k_{\jmath}}{g}} \frac{H^{+}\left(k_{\jmath}\right)}{\sqrt{1-4 \tau}} u(1-4 \tau) & j=1,2 \\ -\sqrt{\frac{k_{\jmath}}{g}} \frac{H^{-}\left(k_{\jmath}\right)}{\sqrt{1+4 \tau}} & j=3,4\end{cases}
$$

Here $H^{ \pm}\left(k_{j}\right)$ is the Kochin function with the following definition

$$
\begin{gathered}
H^{ \pm}\left(k_{j}\right)=\left[\int_{S_{L}}+\int_{S_{R}}\right]\left(\frac{\partial \phi}{\partial n}-\phi \frac{\partial}{\partial n}\right) e^{-k_{j} y \pm i k_{j} x} d l \\
j=\left(\begin{array}{ll}
1, & 2 \\
3, & 4
\end{array}\right)
\end{gathered}
$$

and can be divided linearly in accordance with the linear decomposition of the velocity potential $\phi$, as follows

$$
\begin{aligned}
H^{ \pm}(k) & =\frac{g A}{i \omega_{0}} H_{D^{ \pm}}(k)+\sum_{j=1}^{s} i \omega \epsilon_{j} X_{j} a H_{j}^{ \pm}(k) \\
& \equiv \frac{g A}{i \omega_{0}} H_{F^{ \pm}}(k)
\end{aligned}
$$

where $H_{F^{ \pm}}(k)=H_{D^{ \pm}}(k)-\sum_{j=1}^{3} \frac{\omega \omega_{0} a}{g} \frac{\epsilon_{j} X_{j}}{A} H_{j}^{ \pm}(k)$

From Eqs. (17) and (18), it can be seen that for $\tau<1 / 4$ an oscillating and translating body generates four waves of different wavenumbers $k_{j}(j=1,2,3$, 4). To be more specific, at $x=\infty$ there exists one wave with wavenumber $k_{2}$ (hereafter referred to as $k_{2}$-wave) which propagates upstream, and at $x=-\infty$ there exist $k_{1}$-wave propagating in the positive $x$ direction and $k_{3}$ - and $k_{4}$-waves radiating downstream. For $\tau>1 / 4, k_{1}$ - and $k_{2}$-waves are not generated and thus only two downstream waves with wavenumbers $k_{3}$ and $k_{4}$ exist. The propagating driections and relative wavelengths of the respective waves are schematically shown in Fig.1.

It is noteworthy that when seen from the moving body the $k_{3}$-wave certainly propagates downstream, but in the space-fixed reference frame it propagates in the same direction as the body; that is, the phase velocity of $k_{3}$-wave is less than the forward velocity of the body. Therefore in the diffraction problem, depending on the ratio of the forward velocity of the body $U$ to the phase velocity of the incident wave $C$, there are three types of following waves: $k_{2}$-wave for $U / C<1 / 2$, $k_{1}$-wave for $1 / 2<U / C<1$, and $k_{3}$-wave for $U / C>1$. On the contrary, the head wave is only $k_{4}$-wave irrespective of the forward velocity. One more thing to be noted is that since both $k_{2}$ and $k_{4}$ reduce to $\omega_{0}^{2} / g$ in the limit of $U \rightarrow 0, k_{2}-$ and $k_{4}$-waves are considered to originate from the zero-speed oscillatory waves. By the similar argument, it can be said that $k_{1}$ - and $k_{3}$-waves originate from the steady translation waves.

\section{Hydrodynamic pressure forces}

In this section we briefly summarize the calculation formulae for the added-mass and damping coefficients, wave-exciting forces, and added resistance. More detailed derivations may be found in reference 2 ).

The hydrodynamic forces on the left and right cylinders are independently calculated by the pres- 
sure integration over the respective surfaces. In calculating the pressure on the body surface, the effect of steady perturbation velocity is taken into account. For example the hydrodynamic force on the left cylinder is given by the following equation

$$
\begin{gathered}
F_{i}^{L}=\sum_{j=1}^{8} T_{i j} \epsilon_{i} \epsilon_{j} X_{j} \\
T_{i j}{ }^{L}=\rho a^{2}(i \omega)^{2} \int_{S_{L}}\left(n_{\imath}-\frac{U}{i \omega a} m_{\imath}\right)\left\{\varphi_{j}+\frac{U}{i \omega a} \hat{\varphi}_{j}\right\} d \theta \\
\equiv-(i \omega)^{2}\left[A_{i j}{ }^{L}+B_{i j}{ }^{L} / i \omega\right]
\end{gathered}
$$

where $A_{i j}{ }^{L}$ and $B_{i j}{ }^{L}$ are respectively the addedmass and damping coefficients of the left cylinder associated with the force in the $i$-th direction due to the $j$-th mode of motion. Similar expressions can be obtained for the right cylinder, with the pressure integrated over $S_{R}$. The added mass and damping of a pair of two cylinders are given by

$$
\begin{aligned}
& A_{i j}=A_{i j}{ }^{L}+A_{i j}{ }^{R} \equiv 2 \rho \pi a^{2} \epsilon_{i} \epsilon_{j} A_{i j^{\prime}} \\
& B_{i j}=B_{i j}{ }^{L}+B_{i j}{ }^{R} \equiv 2 \rho \pi a^{2} \epsilon_{i} \epsilon_{j} \omega B_{i j}{ }^{\prime}
\end{aligned}
$$

The damping coefficient can be also derived by applying the principle of energy conservation. Using the Kochin function defined by Eq. (21), the result is expressed in the form ${ }^{2)}$ )

$$
\begin{array}{r}
B_{j j^{\prime}}=\frac{1}{4 \pi}\left[\frac{\left|H_{j}{ }^{+}\left(k_{1}\right)\right|^{2}+\left|H_{j}+\left(k_{2}\right)\right|^{2}}{\sqrt{1-4 \tau}}\right. \\
\left.+\frac{-\left|H_{j}-\left(k_{3}\right)\right|^{2}+\left|H_{j}-\left(k_{4}\right)\right|^{2}}{\sqrt{1+4 \tau}}\right]
\end{array}
$$

The two values of (27) and (28) should be identical. This energy relation is used as a check tool for the accuracy of numerical computations.

The wave-exciting force in the $j$-th direction on the left cylinder can be obtained by the integration of the form

$$
\frac{E_{j}^{L}}{\rho g A a \epsilon_{j}}=\frac{\omega}{\omega_{0}} \int_{S_{L}}\left(n_{j}-\frac{U}{i \omega a} m_{j}\right)\left\{\varphi_{I}+\varphi_{D}\right\} d \theta
$$

If the same integration is done over the surface $S_{R}$, the exciting force on the right cylinder can be obtained. Adding these two values, the total wave-exciting force on a pair of two cylinders is given as

$$
E_{j}=E_{j}{ }^{L}+E_{j}{ }^{R} \equiv 2 \rho g A a \epsilon_{j} E_{j}{ }^{\prime}
$$

This total exciting force can be also calculated in terms of the Kochin function for the radiation problem (Haskind-Newman's relation). That is,

$$
E_{\jmath}^{\prime}=\frac{\omega}{\omega_{0}} \tilde{H}_{\jmath}^{ \pm}(k)=\frac{\omega}{\omega_{0}}(-1)^{\jmath} H_{\jmath}^{ \pm}(k)
$$

where $\tilde{H}_{j}^{ \pm}(k)$ denotes the Kochin function for the reverse-flow radiation problem and, as in Eq. (31), for the body symmetrical about $x=0$ this function is related in a simple form to the original radiation-problem Kochin function $H_{j}^{ \pm}(k)$. The numerical accuracy for the diffraction problem is checked by comparing the two values of Eqs. (30) and (31).
The added resistance, which is the second-order steady force but of practical interest to naval architects, can be calculated by the following equations $^{2)}$ :

1) for $k=k_{4}$ (head wave)

$$
\begin{aligned}
& R_{A W^{\prime}}=\frac{1}{2}\left[\frac{\left|H_{D}{ }^{+}\left(k_{1}\right)\right|^{2}}{\sqrt{1-4 \tau}}\left(1+\frac{k_{1}}{k_{4}}\right)\right. \\
& \left.+\frac{\left|H_{D}{ }^{+}\left(k_{2}\right)\right|^{2}}{\sqrt{1-4 \tau}}\left(1+\frac{k_{2}}{k_{4}}\right)+\frac{\left|H_{D}-\left(k_{3}\right)\right|^{2}}{\sqrt{1+4 \tau}}\left(\frac{k_{3}}{k_{4}}-1\right)\right]
\end{aligned}
$$

2) for $k=k_{2}$ (following wave, $0<U / C<1 / 2$ )

$$
\begin{aligned}
& R_{A W^{\prime}}=\frac{1}{2}\left[\frac{\left|H_{D}+\left(k_{1}\right)\right|^{2}}{\sqrt{1-4 \tau}}\left(\frac{k_{1}}{k_{2}}-1\right)\right. \\
& \left.\quad+\frac{\left|H_{D}-\left(k_{3}\right)\right|^{2}}{\sqrt{1+4 \tau}}\left(1+\frac{k_{3}}{k_{2}}\right)-\frac{\left|H_{D}-\left(k_{4}\right)\right|^{2}}{\sqrt{1+4 \tau}}\left(1+\frac{k_{4}}{k_{2}}\right)\right]
\end{aligned}
$$

3) for $k=k_{1}$ (following wave, $1 / 2<U / C<1$ )

$$
\begin{aligned}
& R_{A W^{\prime}}=\frac{1}{2}\left[-\frac{\left|H_{D}+\left(k_{2}\right)\right|^{2}}{\sqrt{1-4 \tau}}\left(1-\frac{k_{2}}{k_{1}}\right)\right. \\
& \left.+\frac{\left|H_{D}-\left(k_{3}\right)\right|^{2}}{\sqrt{1+4 \tau}}\left(1+\frac{k_{3}}{k_{1}}\right)-\frac{\left|H_{D}-\left(k_{4}\right)\right|^{2}}{\sqrt{1+4 \pi}}\left(1+\frac{k_{4}}{k_{1}}\right)\right]
\end{aligned}
$$

4) for $k=k_{3}$ (following wave, $U / C>1$ )

$$
\begin{aligned}
& R_{A W^{\prime}}=\frac{1}{2}\left[\frac{\left|H_{D}{ }^{+}\left(k_{1}\right)\right|^{2}}{\sqrt{1-4 \tau}}\left(1+\frac{k_{1}}{k_{3}}\right)\right. \\
& \left.+\frac{\left|H_{D}+\left(k_{2}\right)\right|^{2}}{\sqrt{1-4 \tau}}\left(1+\frac{k_{2}}{k_{3}}\right)+\frac{\left|H_{D}-\left(k_{4}\right)\right|^{2}}{\sqrt{1+4 \tau}}\left(1-\frac{k_{4}}{k_{3}}\right)\right]
\end{aligned}
$$

where

$$
R_{A W^{\prime}}=R_{A W} / \frac{1}{2} \rho g A^{2}
$$

The above equations (32)-(35) are for the diffraction problem, but when the body is allowed to oscillate, the Kochin function $H_{D}^{ \pm}(k)$ must be replaced by the motion-free Kochin function $H_{F}^{ \pm}(k)$ defined by Eq. (22).

\section{Numerical results and discussion}

The numerical scheme to solve the integral equation (16) is as follows. The contours of right and left cylinders are discretized with $N_{R}$ and $N_{L}$ segments respectively and the velocity potential is assumed constant on each segment. Then, with the fact that the velocity potential is a complex quantity, Eq. (16) reduces to a linear system of algebraic equations with $2\left(N_{R}+N_{L}\right)$ unknowns; this was solved by the direct matrix-inversion method. Numerical accuracy was checked by the energy-conservation principle and the HaskindNewman relation. With $N_{R}=N_{L}=60$, these relations were confirmed to be satisfied with errors less than $0.5 \%$.

\section{1 Added-mass and damping coefficients}

For comparison, the added mass of a single circular cylinder with depth-to-radius ratio $d / a=2$ is presented in Fig. 2(a). The values of interacting left and right cylinders are shown in Figs. 2 


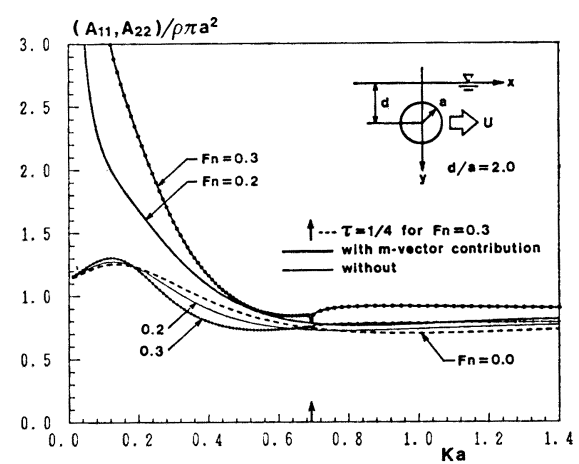

Fig. 2 (a) Added-mass coefficients for surge and heave motions of a single cylinder

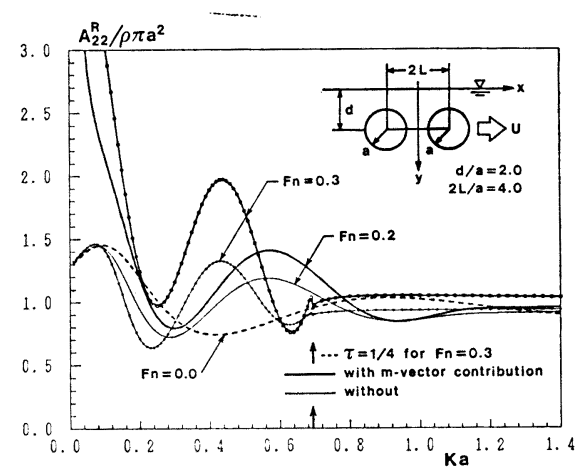

Fig. 2 (c) Heave added-mass coefficient (right cylinder)

(b) and (c) respectively for $d / a=2$ and spacingto-radius ratio $2 L / a=4$. Fig. 2 (d) shows the added mass of a pair of two cylinders which is, as obvious from Eq. (26), given by the arithmetical average of Figs. 2(b) and (c). In these and subsequent figures for radiation forces, the results for Froude numbers $0.0,0.2$, and 0.3 are includ. ed, and for respective Froude numbers two kinds of computations are.performed: one including the effect of steady perturbation velocity (which is displayed as $m$-vector contribution with thick solid lines) and the other ignoring the $m$-vector throughout the calculations (indicated by thin solid lines). Comparison of thick and thin solid lines reveals that the $m$-vector exerts a considerable influence not to be neglected.

The effects of hydrodynamic interaction can be investigated by comparing Fig. 2(a) with Figs. 2 (b) and (c). Remarkable effects can be seen on the right cylinder, particularly in the frequency range lower than the critical frequency $(\tau<1 / 4)$. However for $\tau>1 / 4$, the interaction effects on the right cylinder are markedly diminished and the results are similar to those of a single cylinder. The effects on the left cylinder exist for all frequencies,

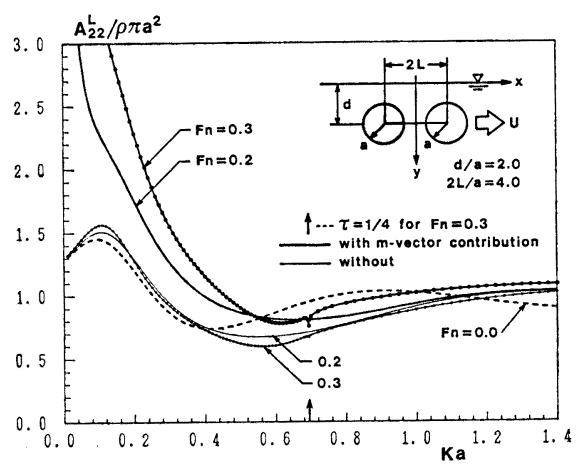

Fig. 2 (b) Heave added-mass coefficient (left cylinder)

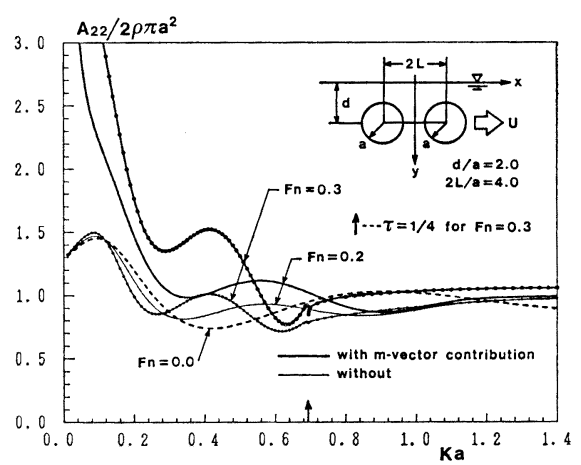

Fig. 2 (d) Heave added-mass coefficient (two cylinders)

but not so large compared with the right cylinder for $\tau<1 / 4$.

These numerical results can be explained qualitatively by the radiated-wave characteristics of a single circular cylinder. As stated in Section 3, when $\tau<1 / 4$, the left cylinder generates the $k_{2}-$ wave propagating toward the right cylinder; this may be viewed as the "following-wave condition" for the right cylinder. On the other hand, the left cylinder is affected by the $k_{1^{-}}, k_{3^{-}}$, and $k_{4^{-}}$ waves generated by the right cylinder and even when $\tau>1 / 4, k_{3}$ - and $k_{4}$-waves have contributions. Taking account of these and that the exciting force in following $k_{2}$-wave varies between zero and a large maximum value as a function of the frequency $^{2)}$, it can be envisaged that for $\tau<1 / 4$ the interaction effects on the right cylinder are large compared to the left cylinder, whereas for $\tau>1 / 4$ the effects become small on the right cylinder but still remain on the left cylinder.

The damping coefficients for the heave motion are shown in Fig. 3 (a) for single cylinder, in Fig. 3 (b) for left cylinder, in Fig. 3(c) for right cylinder, and in Fig. 3 (d) for two cylinders as a unit. By comparison, the interaction effects are 


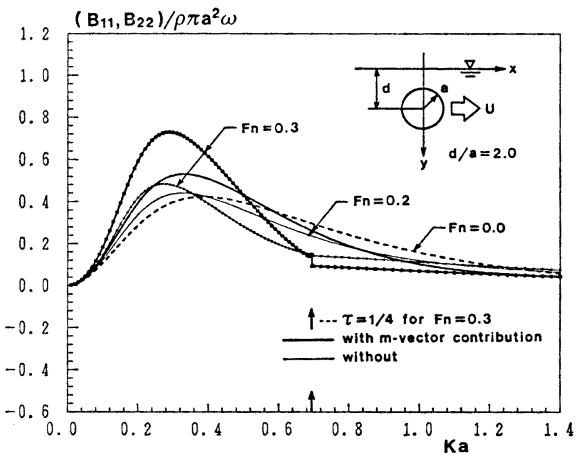

Fig. 3 (a) Damping coefficients for surge and heave motions of a single cylinder

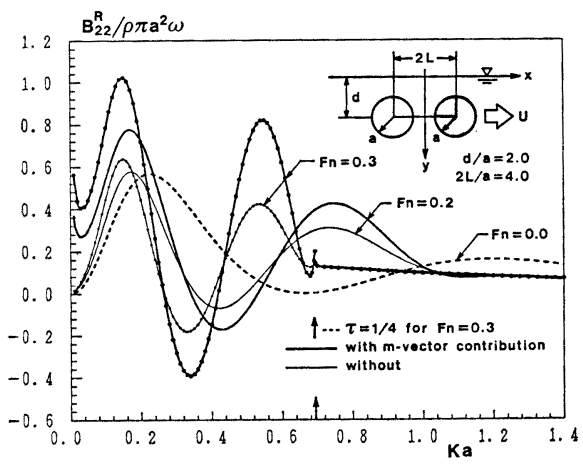

Fig. 3 (c) Heave damping coefficient (right cylinder)

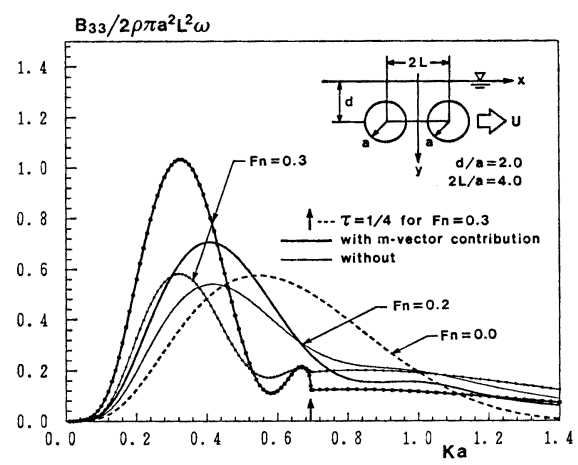

Fig. 4 Damping coefficient for pitch motion of two cylinders

striking on the right cylinder. Considerations on the effects of $m$-vector and hydrodynamic interactions may be the same as that for the added mass. One noteworthy point is that both left and right cylinders experience the negative damping for nonzero Froude numbers. However, as seen in Fig. 3(d), the total damping force of two cylinders is certainly positive.

As an example of the numerical results relating to the pitching motion, we present the damping

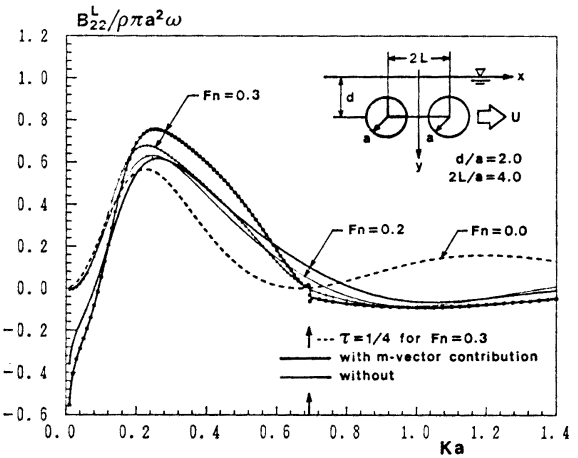

Fig. 3 (b) Heave damping coefficient (left cylinder)

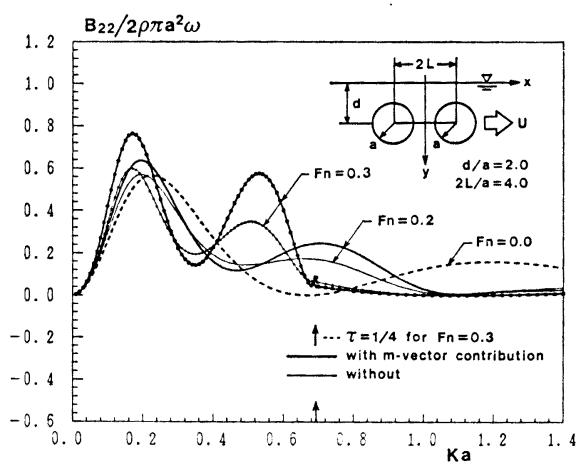

Fig. 3 (d) Heave damping coefficient (two cylinders)

coefficient of pitch $\left(B_{33}\right)$ in Fig. 4. Comparison of $B_{33}$ with $B_{22}$ shown in Fig. $3(\mathrm{~d})$ indicates that the variation of $B_{33}$ due to hydrodynamic interactions is not so remarkable.

\section{2 Wave exciting forces}

Figs. 5 ( a), (b), and (c) show the amplitude of surge exciting force in head waves for single, left, and right cylinders, respectively. By comparison, one can notice that the interaction effects are not so large and that the results of left and right cylinders are almost the same and insensitive to the Froude number.

Physical interpretations for these results are given below, in terms of the diffraction characteristics of a single circular cylinder. We note that, as proved by Grue $\& \mathrm{Palm}^{5)}$, both $k_{1}$ - and $k_{2}$-waves are not generated for the incident $k_{4}$-wave and that the diffracted $k_{3}$-wave is of small amplitude. From these properties, it can be envisaged that the $k_{4}-$ wave retains almost the same amplitude (but possibly experiences a phase shift) when passing the right cylinder and then strikes the left cylinder. In consequence, the amplitudes of exciting forces on left and right cylinders are expected to be nearly equal and not to be so different from that of a single cylinder. 


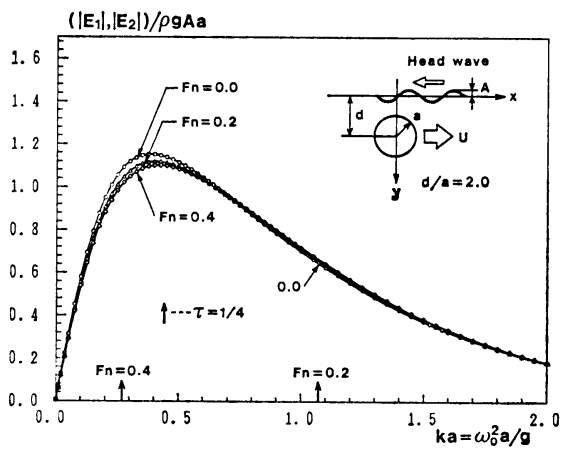

Fig. 5 (a) Exciting forces for surge and heave motions of a single cyl inder in head wave

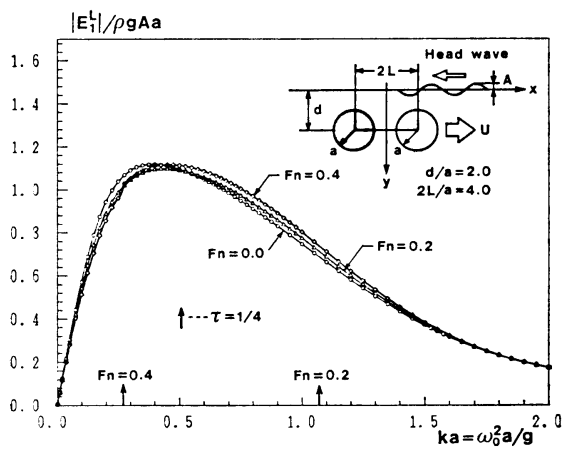

Fig. 5 (b) Surge exciting force in head wave (left cylinder)

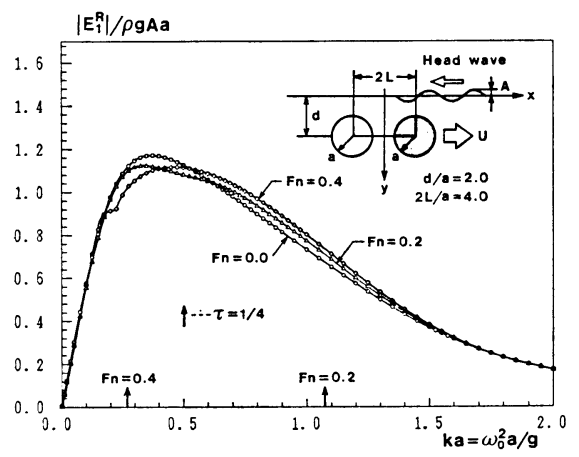

Fig. 5 (c) Surge exciting force in head wave (right cylinder)

Figs. 6 (a)-(c) present the amplitude of surge exciting force in following waves. In the range of frequencies shown in these figures, the wavenumber of incident wave is $k=k_{2}$ for $F_{k}=0.0$ and 0.2 , but for $F_{n}=0.4, k=k_{2}$ for $0<k a<1.5625$ and $k=k_{1}$ otherwise. The frequency corresponding to $k a=1.5625$, indicated by the upward arrow, is the critical frequency where the velocity of the body equals the group velocity of the incident wave,

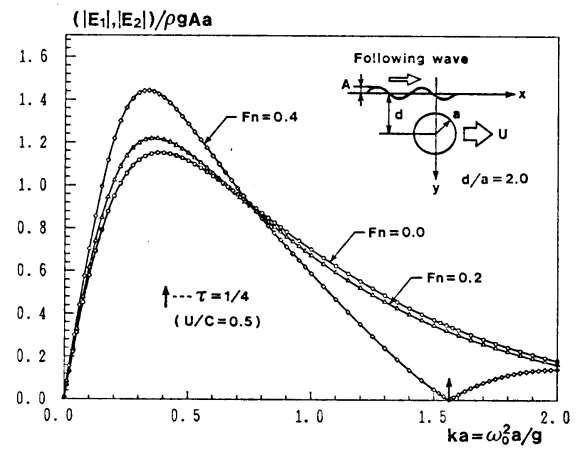

Fig. 6 (a) Exciting forces for surge and heave motions of a single cylinder in following wave

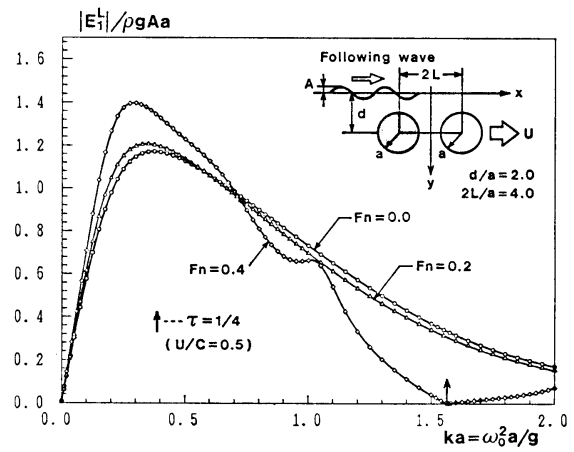

Fig. 6 (b) Surge exciting force in following wave (left cylinder)

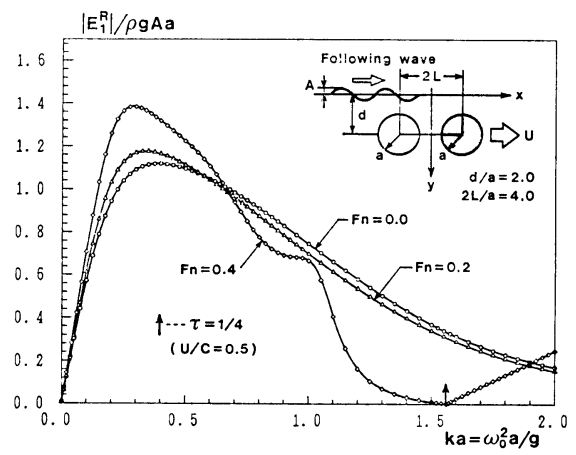

Fig. 6 (c) Surge exciting force in following wave (right cylinder)

thus $\tau=1 / 4$. The vanishing of the exciting forces at this critical frequency is one of the distinctive features in the following-wave problem. Comparing Fig. 6 (b) with (c), we notice that the variation of the left cylinder is analogous to that of the right cylinder; this tendency is the same as the head-wave condition.

A physical interpretation for the above is as follows. When the $k_{2}$-wave is incident upon a mov- 
ing circular cylinder, generated are the waves of wave numbers $k_{1}$ and $k_{2}$ only, with $k_{1}$ - and $k_{2}-$ waves located downstream and upstream, respectively. Considering that the $k_{1}$-wave originates from the steady translation wave, the amplitude of diffracted $k_{1}$-wave is expected to increase gradually from zero (the $k_{2}$-wave correspondingly decreases) with $U / C$ or, equivalently, with incidentwave frequency. Thus in the low frequency range, the $k_{2}$-wave impinging upon the right cylinder remains almost unchanged in amplitude after transmitting the left cylinder; as a result, the exciting forces on the right and left cylinders are almost the same. However as the frequency approaches the critical point $(\tau=1 / 4)$, owing to the decreasing transmitted $k_{2}$-wave, the exciting force on the right cylinder is expected to be reduced compared to that on the left cylinder; this can be confirmed by comparing Fig. 6 (b) with (c). When $\tau>1 / 4$, besides the incident wave ( $k_{1}$-wave), the diffracted $k_{2}$-wave impinges on the right cylinder and then the right cylinder generates the new $k_{1}$-wave downstream, which in turn affects the left cylinder, and so on. As can be imagined from such a consideration, the hydrodynamic interactions for $\tau>1 / 4$ appear to be somewhat complicated.

\section{3 Motions of two rigidly-connected cylin- ders}

We computed the motions of two rigidly-connected cylinders responding to the first-order hydrodynamic forces obtained above. With the assumption that the density of cylinders is the same as that of fluid, the following simultaneous linear equations were used to describe the coupled motions of surge, heave, and pitch.

$$
\sum_{j=1}^{3}\left[-\omega^{2}\left(M_{\imath \delta_{i j}}+A_{i j}\right)+i \omega B_{i j}\right] \epsilon_{j} X_{j}=E_{\imath} \quad(i=1,2,3)
$$

where $\delta_{i j}$ denotes the Kroenecker delta function and $M_{i}$ the mass (for $i=1,2$ ) or the moment of inertia (for $i=3$ ) of the two rigidly-connected cylinders.

As examples of computed results, the absolute value of the "complex" pitching motion $X_{3}$ is pre-

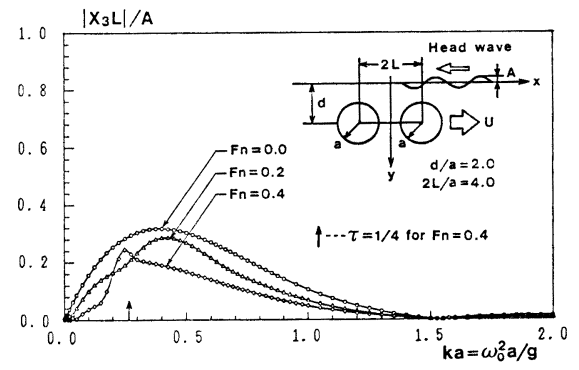

Fig. 7 Amplitude of pitch motion of two cylinders in head wave sented in Fig. 7 for head waves and in Fig. 8 for following waves. It is seen from these figures that in head waves the forward speed affects the body motion to reduce its amplitude, whereas in follow. ing waves the amplitude increases with the increasing Froude number.

\section{4 Added resistance}

The added resistance in head waves turned out to be approximately zero (thus the graph is not

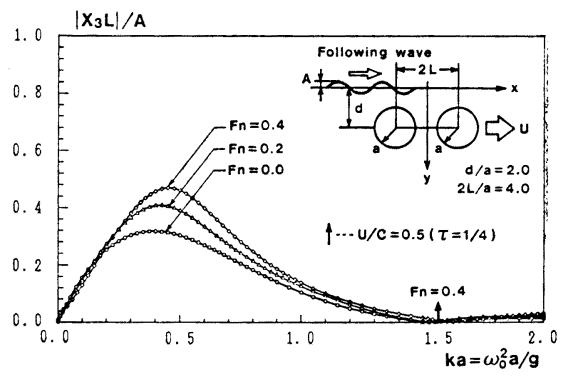

Fig. 8 Amplitude of pitch motion of two cylinders in following wave

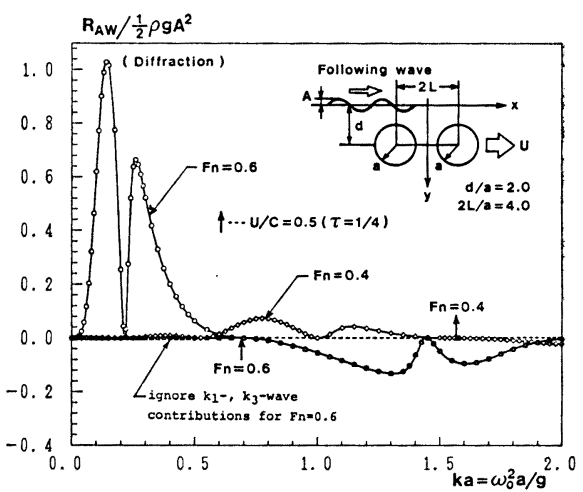

Fig. 9 Added resistance of submerged two cylinders in following wave (diffraction)

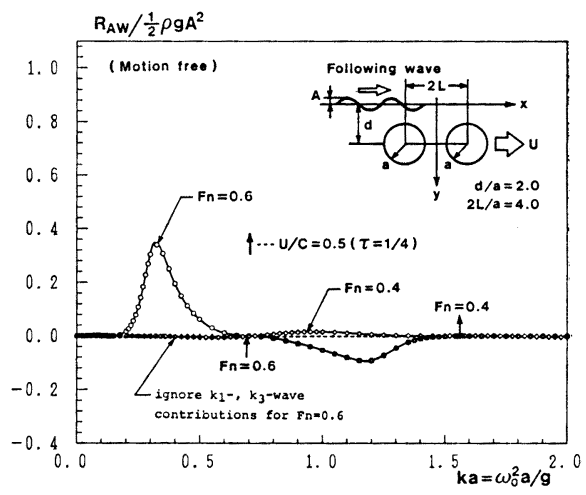

Fig. 10 Added resistance of submerged two cylinders in following wave (motion free) 
presented). This can be explained in the following manner. As discussed in relation to the exciting force, even if the hydrodynamic interactions are taken into account, both $k_{1}$ - and $k_{2}$-waves are not generated for the incident $k_{4}$-wave, and further the amplitude of diffracted $k_{3}$-wave is quite small. Thus, from Eq. (32), the added resistance in head waves is nearly equal to zero for all frequencies.

Fig. 9 shows the added resistance of two rigidlyconnected cylinders restrained in following waves. Fundamental features of this result are the same as those of a single circular cylinder ${ }^{2)}$ ); that is, the added resistance is positive for the $k_{2}$-wave $(U / C<$ $1 / 2)$, but negative for the $k_{1}$-wave $(1 / 2<U / C<$ 1). A remarkable feature is that there are a number of frequencies where the added resistance vanishes owing to the hydrodynamic interactions.

If the body is freely oscillating, all four waves of different wavenumbers $k_{j}(j=1,2,3,4)$ contribute to the added resistance. Using the numerical results of body motions, we calculated the added resistance of unrestrained two cylinders from Eqs. (33) and (34), with $H_{D^{ \pm}}(k)$ replaced by $H_{F}^{ \pm}(k)$ in Eq. (22). The result is shown in Fig. 10. Comparing Fig. 10 with Fig. 9, we see that the effects of body motions fairly diminish the added resistance and that there is no drastic change due to hydrodynamic interactions.

\section{Concluding remarks}

Under a linear assumption, we calculated exactly the hydrodynamic forces acting on the two-dimensional two circular cylinders, moving with forward velocity and oscillating in head and following waves. Then we investigated the effects of hydrodynamic interaction on the radiation forces, diffraction forces, and added resistance.

Principal knowledge obtained through the present study can be summarized as follows :

(1) In the low frequency range $(\tau<1 / 4)$, the hydrodynamic interactions exert a great influence on the radiation forces of the right (upstream) cylin. der, whereas for $\tau>1 / 4$ this influence is markedly reduced. The left (downstream) cylinder is affected by the hydrodynamic interactions irrespective of the value of $\tau$, but not so considerably as compared with the right cylinder for $\tau<1 / 4$.

(2) Both left and right cylinders experience the negative damping for nonzero forward velocity, but the total damping on two cylinders as a unit is certainly positive.

(3) The hydrodynamic interaction effects on exciting forces are not so large in comparison with the radiation forces.

(4) The added resistance of two rigidly-connected circular cylinders is approximately zero in head waves. In following waves, it is drastically changed by the interaction effects, while retaining the positive value for $U / C<1 / 2$ and the negative value for $1 / 2<U / C<1$.

These numerical results are qualitatively in agreement with the physical consideration using the radiated- and diffracted-wave characteristics of a single circular cylinder.

\section{Acknowledgements}

The authors would like to thank Professor M. Ohkusu of Research Institute for Applied Mechanics, Kyushu University for his helpful suggestions and encouragement in the course of this study.

This research is partly supported by the Grantin-Aid for Scientific Research of the Ministry of Education, Science and Culture.

\section{References}

1) Kagemoto, H. and Yue, D. K. P.: hydrodynamic Interaction among Multiple Three $\mathrm{Di}$ mensional Bodies: An Exact Algebraic Method, J. Fluid Mech., Vol. 166 (1986), 189-209.

2) Kashiwagi, M., Varyani, K. and Ohkusu, M.: Forward-speed Effects on hydrodynamic Forces Acting on a Submerged Cylinder in Waves, Rept. of Res. Inst. Applied Mech., Kyushu Univ., Vol. 34 (1987), (to be publish. ed).

3) Kashiwagi, M., Varyani, K. and Ohkusu, M.: Hydrodynamic Forces Acting on a Submerged Elliptic Cylinder Translating and Oscillating in Waves, Trans. of West-Japan Society of Nav. Arch., Vol. 74 (1987), (in press).

4) Ohkusu, M.: Hydrodynamic Forces on Multiple Cylinders in Waves, Proc. Intl. Symp. on Dynamics of Marine Vehicles and Structures in Waves, London, Paper 12 (1974), 107-112.

5) Grue, J. and Palm, E.: Wave Radiation and Wave Diffraction from a Submerged Body in a Uniform Current, J. Fluid Mech., Vol. 151 (1985), 257-278.

\section{Appendix $m$-vector for submerged two circular cylinders}

The calculation of $m$-vector requires a solution for the steady perturbation potential $\varphi_{8}$. To avoid numerical difficulties, we use the infinite-fluid solution valid for a "deeply" submerged body.

Let us consider two cylinders, composed of the left circular cylinder of radius $a$ (referred to as circle $\mathrm{L}$ ) and the right circular cylinder of radius $b$ (circle R), with separation distance between cylinder centers $c$ and angle of inclination $\alpha$ (see Fig. A 1). These two cylinders are assumed to 


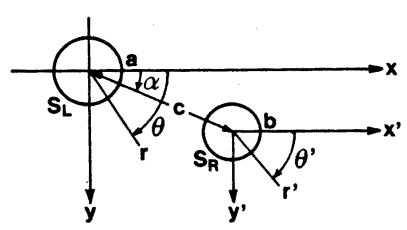

Fig. A 1 Coordinate system for the $m$-vector calculation

move with forward speed $U$ in the positive $x$-direction. We express the velocity potential as

$$
\varphi_{\mathrm{s}}=U\left(\varphi^{L}+\varphi^{R}\right) \text {. }
$$

Then the body boundary conditions to be satisfied by $\varphi^{L}$ and $\varphi^{R}$ take the following form

$$
\left\{\begin{array}{lll}
\frac{\partial \varphi^{L}}{\partial r}=\cos \theta & \frac{\partial \varphi^{R}}{\partial r}=0 & \text { on circle } L \\
\frac{\partial \varphi^{L}}{\partial r^{\prime}}=0 & \frac{\partial \varphi^{R}}{\partial r^{\prime}}=\cos \theta^{\prime} & \text { on circle } R
\end{array}\right.
$$

Physically $\varphi^{L}$ describes the flow, in which only circle $\mathrm{L}$ moves, with circle $\mathrm{R}$ at rest. Similarly $\varphi^{R}$ is for the flow where only circle $R$ moves, with circle $\mathrm{L}$ at rest.

First let us consider $\varphi^{L}$. If circle $R$ were not present, the flow past circle $L$ could be represented by a point doublet located at the origin. The change caused by the presence of restrained circle $R$ is represented by the image doublet at the "mirrorimage" point inside circle $R$, and in turn the effect of this image doublet on circle $L$ is taken into account by introducing the image doublet inside circle L. As a result of repeating such procedures, $\varphi^{L}$ is given by an infinite series of image doublets within circle $\mathrm{L}$ and circle $\mathrm{R}$. With the complex velocity potential, it can be written in the following form

$$
f^{L}(z)=-a \sum_{j=0}^{\infty} \frac{\mu_{j} e^{i \beta_{j}}}{z-\gamma_{j} e^{i \alpha}}, z=r e^{i \theta}
$$

where

$$
\begin{aligned}
& \gamma_{0}=0 \quad r_{2 n-1}=r-\frac{\varepsilon^{2}}{\gamma-\gamma_{2 n-2}} \quad r_{2 n}=\frac{1}{\gamma_{2 n-1}} \\
& \mu_{0}=1 \frac{\mu_{2 n-1}}{\mu_{2 n-2}}=\left(\frac{\varepsilon}{\gamma-\gamma_{2 n-2}}\right)^{2} \frac{\mu_{2 n}}{\mu_{2 n-1}}=\left(\frac{1}{\gamma_{2 n-1}}\right)^{2} \\
& \beta_{0}=0 \quad \beta_{2 n-1}=2 \alpha-\pi \quad \beta_{2 n}=0 \\
& \gamma=c / a, \varepsilon=b / a \quad(n=1,2, \cdots \infty)
\end{aligned}
$$

Likewise, $\varphi^{R}$ can be represented by an infinite series of image doublets, with the leading term being a point doublet located at the origin of circle R. In the coordinate system with the origin at the center of circle $L, \varphi^{R}$ is given as the real part of the complex potential of the form

$$
f^{R}(z)=-a \sum_{j=0}^{\infty} \frac{\mu_{j} e^{i \beta_{j^{\prime}}}}{z-\gamma_{j} e^{i \alpha}}
$$

where

$\gamma_{0}{ }^{\prime}=1 \quad \gamma_{2 n-1}{ }^{\prime}=\frac{1}{\gamma_{2 n-2}{ }^{\prime}} \quad \gamma_{2 n^{\prime}}=\gamma-\frac{\varepsilon^{2}}{\gamma-\gamma_{2 n-1}{ }^{\prime}} \quad$

$$
\left.\begin{array}{ccc}
\mu_{0}^{\prime}=\varepsilon^{2} & \frac{\mu_{2 n-1}{ }^{\prime}}{\mu_{2 n-2}{ }^{\prime}}=\left(\frac{1}{\gamma_{2 n-2}{ }^{\prime}}\right)^{2} & \frac{\mu_{2 n^{\prime}}}{\mu_{2 n-1}{ }^{\prime}}=\left(\frac{\varepsilon}{\gamma-\gamma_{2 n-1^{\prime}}}\right)^{2} \\
\beta_{0}=0 & \beta_{2 n-1}{ }^{\prime}=2 \alpha-\pi & \beta_{2 n^{\prime}=0} \\
(n=1,2, \cdots \infty)
\end{array}\right\}
$$

To calculate the $m$-vector, we consider a typical complex potential given by

$$
f(z)=-a \frac{\mu e^{i \beta}}{z-\gamma e^{i \alpha}}
$$

Substituting this into the definition of the $m$ vector, Eq. (5), the $m$-vector can be readily obtained with the result

$$
\begin{gathered}
m_{1}-i m_{2}=-\frac{\partial}{\partial n}(u-i v)=-\left.\frac{\partial}{a \partial r} f^{\prime}(z)\right|_{r=1} \\
=2 \mu\left\{\frac{1-\gamma e^{i(\theta-\alpha)}}{1-2 \gamma \cos (\theta-\alpha)+\gamma^{2}}\right\}^{8} e^{-i(2 \theta-\beta)}
\end{gathered}
$$

Since the $m$-vector for circle $\mathrm{L}$ is calculated from the sum of Eqs. (A 4) and (A 6), Eq. (A 9) deduces the following results :

$$
\begin{aligned}
m_{1}{ }^{L}= & 2 \sum_{j=0}^{\infty}\left[\mu_{j}\left\{P_{j} \cos \left(2 \theta-\beta_{j}\right)+Q_{j} \sin \left(2 \theta-\beta_{j}\right)\right\}\right. \\
& \left.+\mu_{j}\left\{P_{j}{ }^{\prime} \cos \left(2 \theta-\beta_{j}{ }^{\prime}\right)+Q_{j} \sin \left(2 \theta-\beta_{j}\right)\right\}\right] \\
m_{2}{ }^{L}= & 2 \sum_{j=0}^{\infty}\left[\mu_{j}\left\{P_{j} \sin \left(2 \theta-\beta_{j}\right)-Q_{j} \cos \left(2 \theta-\beta_{j}\right)\right\}\right. \\
& \left.+\mu_{j}\left\{P_{j} \sin \left(2 \theta-\beta_{j}\right)-Q_{j} \cos \left(2 \theta-\beta_{j}{ }^{\prime}\right)\right\}\right]
\end{aligned}
$$

where

$$
\begin{aligned}
& \left.P_{j}=F\left(r_{j}\right), \quad Q_{j}=G\left(r_{j}\right)\right\} \\
& \left.P_{\jmath}^{\prime}=F\left(\gamma_{j}^{\prime}\right), \quad Q_{j}^{\prime}=G\left(\gamma_{\jmath}\right)\right\} \\
& {\left[\begin{array}{l}
F(\gamma) \\
G(\gamma)
\end{array}\right]=\sum_{k=0}^{3} a_{k} \gamma^{k}\left[\begin{array}{l}
\cos k(\theta-\alpha) \\
\sin k(\theta-\alpha)
\end{array}\right]} \\
& /\left\{1-2 \gamma \cos (\theta-\alpha)+\gamma^{2}\right\}^{3} \\
& a_{0}=1, a_{1}=-3, a_{2}=3, a_{3}=-1
\end{aligned}
$$

Next we proceed to the calculation of $m$-vector for circle $R$. The only necessary task is to rewrite Eqs. (A 4) and (A 6) in the coordinate system with the origin at the center of circle R. Denoting the complex variable in this new coordinate system by $z^{\prime}$, the relation between $z^{\prime}$ and $z$ is

$$
z=z^{\prime}+\gamma e^{i \alpha}
$$

On the surface of circle $R$, we should put $z^{\prime}=\varepsilon e^{i \theta}$. With these taken into account, the $m$-vector for circle $R$ can be easily derived from that for circle $L$ obtained above. The results are expressed in the form

$$
\begin{aligned}
& m_{1}{ }^{R}=\frac{2}{\varepsilon^{3}} \sum_{j=0}^{\infty}\left[\mu_{j}\left\{R_{j} \cos \left(2 \theta-\beta_{\jmath}\right)+S_{j} \sin \left(2 \theta-\beta_{j}\right)\right\}\right. \\
& \left.+\mu_{j}\left\{R_{j} \cos \left(2 \theta-\beta_{j^{\prime}}\right)+S_{j} \sin \left(2 \theta-\beta_{j^{\prime}}\right)\right\}\right] \\
& m_{2}{ }^{R}=\frac{2}{\varepsilon^{3}} \sum_{j=0}^{\infty}\left[\mu_{j}\left\{R_{j} \sin \left(2 \theta-\beta_{j}\right)-S_{j} \cos \left(2 \theta-\beta_{j}\right)\right\}\right. \\
& \left.+\mu_{j}\left\{R_{j} \sin \left(2 \theta-\beta_{j^{\prime}}\right)-S_{j^{\prime}} \cos \left(2 \theta-\beta_{j^{\prime}}\right)\right\}\right]
\end{aligned}
$$

where

$$
\left.\begin{array}{l}
R_{\jmath}=F\left(\delta_{j}\right), \quad S_{j}=G\left(\delta_{j}\right) \\
R_{j}=F\left(\delta_{\jmath}\right), \quad S_{\jmath}=G\left(\delta_{\jmath}\right)
\end{array}\right\}
$$




$$
\delta \jmath=\frac{1}{\varepsilon}\left(\gamma_{j}-\gamma\right), \quad \delta j^{\prime}=\frac{1}{\varepsilon}\left(\gamma_{j}^{\prime}-\gamma\right)
$$

Taking account of that the rotational component $m_{3}$ about the center of a circular cylinder is identically zero, we can calculate $m_{3}$ for the two rigidly-connected circular cylinders by the following equations

$$
\left.\begin{array}{l}
m_{3}{ }^{L}=-\left(m_{2}{ }^{L} \cos \alpha-m_{1}{ }^{L} \sin \alpha\right) \\
m_{3}{ }^{R}=\left(m_{2}{ }^{R} \cos \alpha-m_{1}{ }^{R} \sin \alpha\right)
\end{array}\right\}
$$

In practical computations the infinite series are truncated to $M$ mirror images. It is found that $M=10$ suffices to converge the series even for the relatively small separation distance. 